\title{
Upper Garment 3D Modeling for Pattern Making
}

\author{
Jun Zhang ${ }^{1}$, Noriaki Innami ${ }^{2}$, KyoungOk Kim ${ }^{3}$, Masayuki Takatera ${ }^{3}$ \\ ${ }^{1}$ Interdisciplinary Graduate School of Science and Technology, Shinshu University, Ueda, Japan.) \\ ${ }^{2}$ Graduate School of Science and Technology, Shinshu University, Ueda, Japan) \\ ${ }^{3}$ Division of Kansei and Fashion Engineering, Institute for Fiber Engineering (IFES), \\ Interdisciplinary Cluster for Cutting Edge Research (ICCER), Shinshu University, Ueda, Japan) \\ takatera@shinshu-u.ac.jp
}

\section{Purpose}

The aim of this study is to produce an upper garment model for three-dimensional (3D) pattern making. This model will take into account ease allowance and silhouette, and will be used to propose a size-changing method.

\section{Design/methodology/approach}

We used two real garment bodices with a surface suitable for pattern development. The garments were fitted to a designated dummy body and scanned. Using the scanned data, we made those upper garment basic models suitable for 3D pattern making. Using one model, we produced two bodice patterns, one with the original seam lines and the other with seam lines that differed from the original ones, and then compared them with the original jacket bodice. To construct garment models that were different in size from the basic model, we calculated multiplication factors of cross-sectional dimensions (in the front, back, and lateral directions) between the basic garment and the actual garment shape worn on a body for each basic model. Using the multiplication factors, we made two different size garment models from two different size dummies for each basic model. We used these models to make patterns and garments.

\section{Findings}

The reproduced jackets had similar shapes, silhouettes and ease allowances to the original jacket. Two garments of different sizes for each original jacket were made using the multiplication factors, and these garments also had similar silhouettes to the original jacket.

\section{Research limitations/implications}

The implications of the work could be the new size-changing method.

\section{Originality/value}

Using our modeling method, we were able to make complex new garment models that take into account ease allowance and silhouette. The ability to size these models up or down using multiplication factors could be a substitute for the grading method.

\section{Keywords}

Pattern making, 3D garment model, Ease allowance, Size change, Multiplication factor

\section{Introduction}

Nowadays, consumers usually select their clothing from numerous ready-to-wear garments, each produced in several sizes. To meet the ease allowance requirements of these garments, grading is used to increase or decrease the pattern sizes, based on the average difference between sizes. Although this is a convenient way to develop different pattern sizes, it still poses a 'fit' 
problem for individuals. The method does not reflect individual body shapes, so the garments will not be a perfect fit to an individual. It is possible to solve this problem using the draping method, with a dummy model that corresponds to a specific customer. However, this technique is not used for mass production because it is expensive and time-consuming.

To solve this problem, three-dimensional (3D) pattern-making methods have been investigated by many researchers, both geometrically (Cho et al., 2005; Cho et al., 2006a; Zhang et al., 2000) and mechanically (Imaoka, 1989). 3D body data has been used to make garment models and patterns by various 3D pattern-making systems (Watanabe, 1999; Bigliani et al., 2000; Kim and Park, 2004).

In previous studies, researchers used body modelers to make an individual model, (Cho et al., 2006a; Cho et al., 2006b; Takatera and Kim, 2013; Daanen et al., 2008). By transforming a body model with measurement parameters, they developed a computerized pattern-making system for individual body shape. A skirt and upper garment with minimum allowance were successfully made using this system (Cho et al., 2010; Chan et al., 2005).

In 3D pattern-making systems, patterns are developed from garment models. The garment model is formed from a scanned dummy or made by a geometrical modeler. For tight-fitting garments, these models are constructed by smoothing a scanned body (Jeong et al., 2007; Kim et al., 2010; Hlaing et al., 2013; Troynikov and Ashayeri, 2011) or dummy model (Yang and Zhang, 2007; Mahnic and Petrak, 2013), and work well for tight-fitting garments in sport and medical applications. Unfortunately these models have no appropriate ease allowance, and as such are only adapted for use in tight-fitting clothing. In addition they do not work well for formed clothing with ease allowance, such as jackets.

Body modelers have been included in recent 3D apparel computer-aided design (CAD) systems. Practical applications of computerized 3D pattern-making have been developed and used in education and demonstration (For more information see http://www.digitalfashion.jp and http://yuka-alpha.com). Basic garment patterns without ease allowance can be easily obtained by developing the surface of a human body model or dummy model using these CAD systems. However, it should be noted that ease allowance is necessary for garment comfort, movement and design. Even though it is possible for a commercial modeler to make an appropriate garment model with ease allowance, these are difficult to produce.

Some researchers have measured 3D ease allowance, used it for pattern-making and comfort evaluation (Wang and Newton, 2007; Chen et al., 2008; Thomassey and Bruniaux, 2013). However, these studies did not actually produce a 3D garment model. Clothing usually has an uneven ease allowance, depending on its silhouette and design. The silhouette and the shape of clothing produced from patterns developed from those models can be changed after sewing.

It is important to take into account ease allowance when considering size development in pattern making. To obtain different sizes of garment patterns for different size bodies, grading in two dimensions or three dimensions has been used to generate basic upper garment patterns of different sizes (Bye et al., 2008; Wang and Huang, 2011), but it was still difficult to produce a suitable ease allowance when taking into account the real garment shape and style. A 3D garment model that includes an appropriate ease allowance, reflects real garment shape and can be easily deformed for different sizes is therefore necessary for 3D pattern-making.

The aim of this study is to make an upper garment model for 3D pattern-making that takes into account both ease allowance and silhouette. We propose a new modeling method for upper garments, one that can be developed by computerized pattern-making systems. We also propose a magnification method using multiplication factors to make different sized models with appropriate ease allowance.

\section{Modeling Method for Upper Garments}

In this study, the 3D surface data of a jacket were used to make an upper garment model and subsequent patterns. We targeted the jacket and made a jacket bodice without a collar and sleeves, 
then placed the bodice on a female dummy and scanned the shape. The shape measurements were converted to a cross-sectional line model. We used a convex hull for each cross-section to remove dents in the horizontal cross-section. The line model was converted into a polygon model, which was used as the basic garment model. Seam lines were obtained in the 3D scanning process.

\section{Deformation of the Model for Different Sizes}

To construct a garment model suitable for use with different sizes, we developed a new method that creates a garment model by expanding a body model with different multiplication factors. We obtained horizontal multiplication factors between the cross-sections of the basic garment model and the body on which we put the jacket. The multiplication factors were obtained in the front, back, and lateral directions. We made a garment model for different body sizes by deforming the body shape using the multiplication factors.

Figure 1 shows the anatomical body planes and coordinate axes. The planes were defined as follows,

$y z$ plane: a vertical plane through the center front and center back (Sagittal plane)

$x y$ plane: a vertical plane across the shoulder points (Coronal plane)

$x z$ plane: a horizontal plane across the center neck points

The coordinate origin is the crossover point of the three planes.

We measured the distance from the origin to contour in the $y,+z$ and $-z$ directions for each cross-section of the jacket and the body, as shown in Figure 2. $z_{i f}$ and $z_{j b}$ represent the distance from the origin to the jacket contour in the front and back directions, respectively. $z_{b f}$ and $z_{b b}$ represent the distance from the origin to the body contour in the front and back directions, respectively. $x_{j}$ and $x_{b}$ are the widths of a cross-section along the $x$-axis of the jacket and the body, respectively.

The multiplication factors of the dummy body and jacket were calculated using the distances. The multiplication factor in the front direction, $S_{f}$, was determined by $z_{j j} / z_{b} f$, the multiplication factor of the back direction, $S_{b}$, was determined by $z_{j b} / z_{b b}$; and the multiplication factor of the transverse direction, $S_{s}$, was determined by $x_{j} / x_{b}$. Multiplication factors were obtained for each horizontal cross-section.

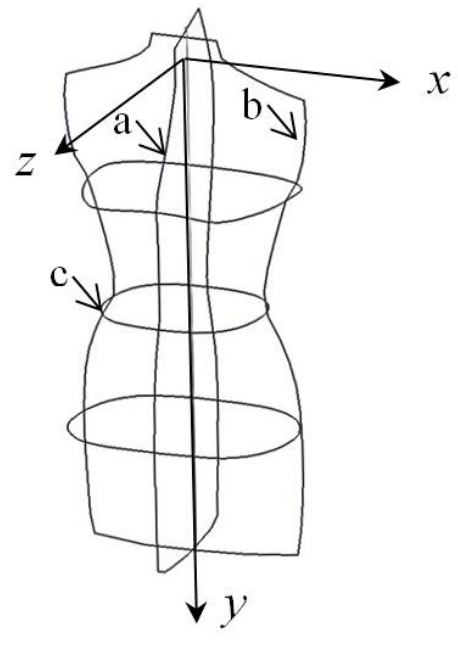

Figure 1. Anatomical body planes (a: Sagittal plane, b: Coronal plane, c: Transverse plane)

To obtain garment models for different body sizes, we enlarged the body using the obtained multiplication factors. For each cross-section of the body, the $z$-coordinates were multiplied by $S_{f}$ if $z<0$, otherwise they were multiplied by $S_{b}$. The $x$-coordinates of all points were multiplied by $S_{s}$. 


\section{Covering and flatting method for pattern making}

After constructing a 3D garment model, covering and flatting methods in 3D pattern-making system (Cho et al., 2006a; Cho et al., 2010) were used to flat 3D surface to 2D patterns. On a 3D garment model surface as shown in Figure 3 (a), two intersecting lines are determined in advance for covering and flatting path. They are equivalent to warp and weft directions in the fabric lattice and named grainlines as shown in Figure 3 (b). The intersection of the primary weft and warp grainlines are the basic reference point used in covering process. We form a fabric lattice with a mesh structure in weft and warp direction. The fabric lattice covers the garment model geometrically with shear from the intersection point of the grainlines as shown in Figure 3 (c). The different grainlines are set in different areas for creating multiple pattern panels.

After the covering, the fabric lattice is cut by arbitrary cutting lines in three dimensions. A 2D pattern is then flatted by returning the shear deformation of the covered lattice. Finally we can obtain 2D pattern as shown in Figure 3 (d).
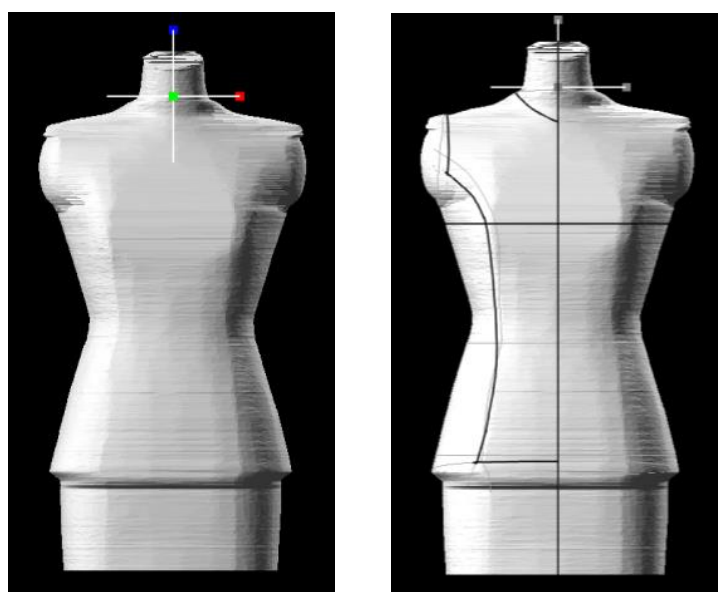

(a) polygon model surface (b) grainlines and cutting lines
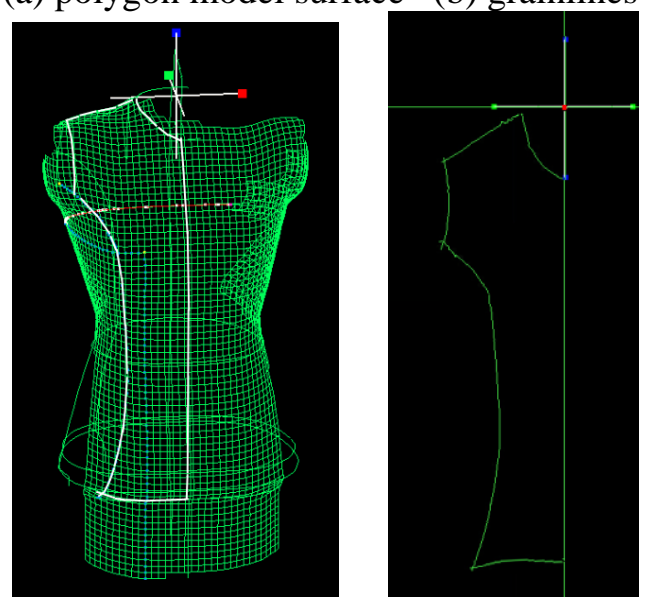

(c) covering

(d) flatting

Figure 3. Covering and flatting method for pattern making

\section{Experimental}

To verify the modeling method, we created two jacket bodice models and reproduced those patterns. The models were also used to make jackets in different sizes, to confirm the proposed deformation method. 
For the experiments, two jackets (Jacket1 and Jacket2) with different patterns were selected to make basic garment models. They were suitable for a Japanese Industrial Standards (JIS) size 9AR female body. The sizes of the body are shown in Table 1. Photographs and patterns of the two jackets are shown in Figures 4 and Figure 5.

In this study, we focused on the bodice part of the jacket. We revised the lapel and the front edge of the original patterns, and also removed the sleeves and collar parts, as shown in Figure 4(c) and Figure 5(b). We carefully made the jacket bodices so that they accurately reflected the original shapes. Cream-colored wool fabric (fabric weight: $149.0 \mathrm{~g} / \mathrm{m}^{2}$ ) was used to reduce scanning errors. To obtain a similar shape to the original, adhesive interlinings (fabric weight: $72.8 \mathrm{~g} / \mathrm{m}^{2}$ ) were fused on every part. The reproduced jacket bodices of Jacket1 and Jacket2 were shown in Figure 6 and Figure 7.

The jacket bodices were worn on size 9AR female body (Kiiya Co., Ltd., Tokyo, Kypris 9AR size) and were scanned by a 3D scanner (Hamamatsu Photonics K.K., Shizuoka, Body Line Scanner). Before scanning, we put small dots of reflective tape on the seam lines of the jacket bodice surface so as to detect the original seam lines in 3D. The scanned data were used to construct basic models by the previously discussed method.

Jackets were constructed from patterns that were created by our model and the seam lines were used as basic verification. To make these patterns, a weft grainline was set on the bust line, and warp grainlines were set orthogonal to the weft grainline. The jacket polygon model was covered by mesh with shear deformation. We set the cutting line according to the detected seam lines. We then used our model to obtain patterns, a process that was performed by the patternmaking system by Cho (Cho et al., 2006a) and Takahashi (Takahashi et al., 2013). After making the jackets, we compared the patterns and jackets with the originals. We also created another patterns and jackets with different cutting lines to confirm the applicability of the 3D model.

To verify the proposed deforming method, we made different size garment models. We selected two female bodies (Kiiya Co., Ltd., Tokyo, Kypris sizes 5AR and 13AR). The measurements of the bodies are shown in Table 1. We obtained the applicable multiplication factors using the basic model and the 9AR body data, with a $2.5 \mathrm{~mm}$ interval. Using the multiplication factors, we expanded the body model of sizes 5AR and 13AR to produce two jacket bodice models. We obtained patterns by applying the garment model to the covering and flatting process. The weft and warp grainlines were set at the same position with the basic model. The cutting lines were set in similar positions to the original jacket.

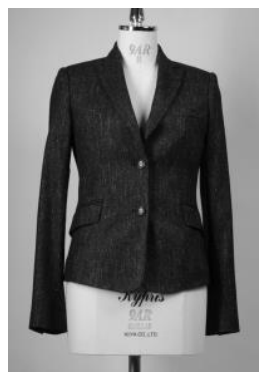

(a)

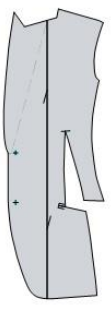

(b)

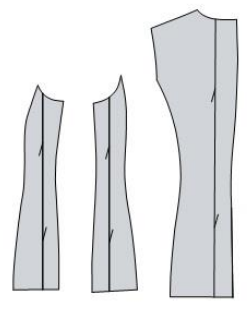

b)

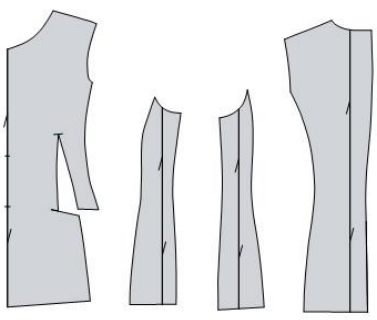

(c)

Figure 4. Jacket1 and patterns for basic model; (a) original jacket, (b) original pattern and (c) simplified pattern 


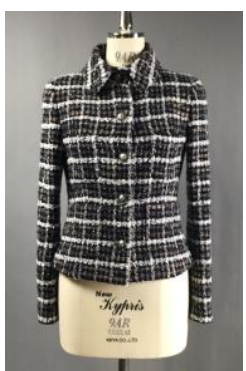

(a)
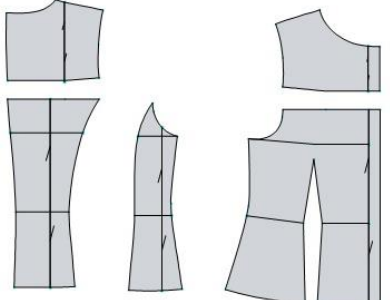

(b)

Figure 5. Jacket2 and patterns for basic model; (a) original jacket, (b) original pattern

Table 1. Measurements for each body size

\begin{tabular}{|l|l|l|l|}
\hline \multicolumn{1}{|c|}{ Body } & \multicolumn{1}{|c|}{ 5AR } & \multicolumn{1}{c|}{ 9AR } & $13 \mathrm{AR}$ \\
\hline $\begin{array}{l}\text { Assumed } \\
\text { height }\end{array}$ & $158 \mathrm{~cm}$ & $158 \mathrm{~cm}$ & $158 \mathrm{~cm}$ \\
\hline Bust & $81 \mathrm{~cm}$ & $87 \mathrm{~cm}$ & $93 \mathrm{~cm}$ \\
\hline Waist & $57 \mathrm{~cm}$ & $63 \mathrm{~cm}$ & $69 \mathrm{~cm}$ \\
\hline Hip & $89 \mathrm{~cm}$ & $93 \mathrm{~cm}$ & $97 \mathrm{~cm}$ \\
\hline Picture & & & \\
\hline
\end{tabular}

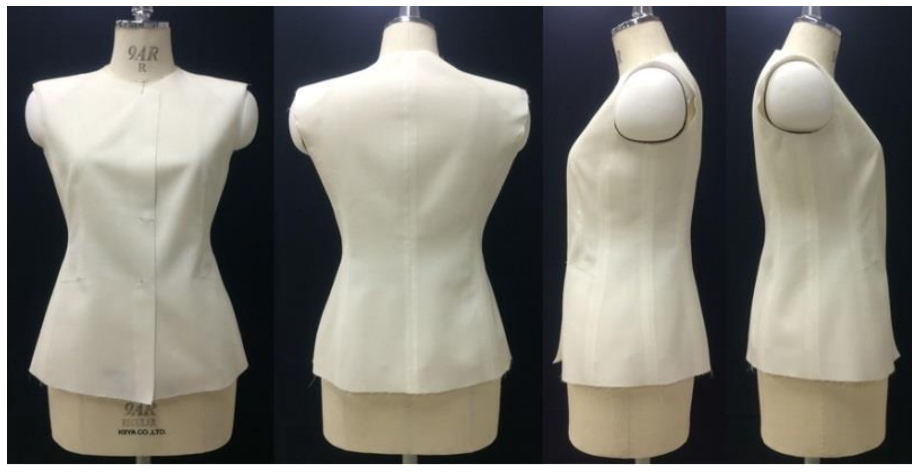
(a) front
(b) back
(c) left side (c) right side

Figure 6. Basic jacket bodice of Jacket 1 


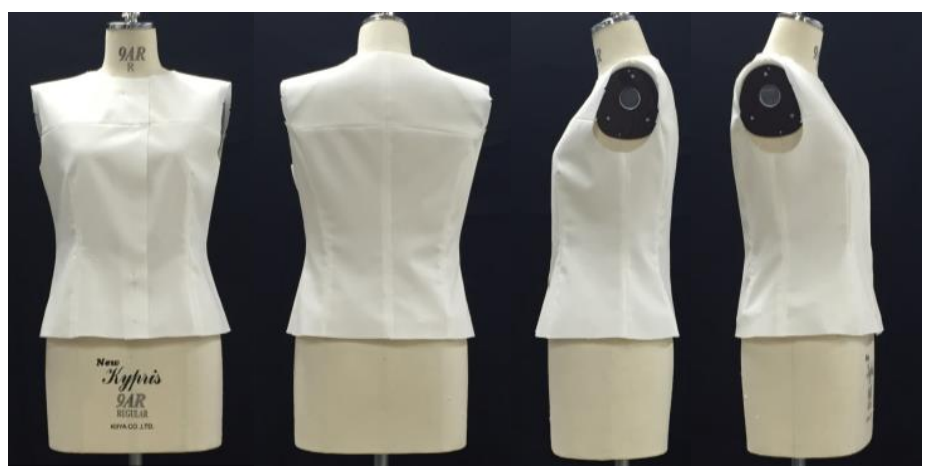

(a) front

(b) back

(c) left side

(c) right side

Figure 7. Basic jacket bodice of Jacket2

\section{Results and Discussion}

The basic garment model of Jacket1 was constructed as demonstrated in Figure 8. Figure 9 shows the configurations of the original cutting lines and the cutting lines that differed. We used different cutting lines to construct three panels instead of the four panels in the original garment. By developing the model according to the cutting lines, we obtained two sets of patterns. Because of restrictions in our system, we obtained separated bodice parts of four segments, as shown in Figure 10 (a). To make patterns that were similar to the original ones, we combined and cut those segments as shown in Figures 10(b) and (c). The final patterns are shown in Figure 10 (d). This process is the same as dart manipulation in pattern-making (Joseph-Armstrong, 2009).

We made two sets of patterns and jackets using Jacket1 bodice model. Figure 11 (a) shows patterns with the same cutting lines as the original. The pattern shapes of the original cutting line were almost the same as the original in Figure 4 (c). Figure 11 (b) shows the jacket made with the same seam lines. It shows almost the same shape and silhouette as the original. Thus, the original pattern and jacket were able to be recreated using the basic garment model.

Figure 12 (a) shows patterns using different cutting lines. The jacket shown in Figure 12 (b) has a similar shape to the original, even though it has fewer panels. It was therefore possible to make a similar garment to the original using the basic garment model with different cutting lines. This indicates that various jacket patterns for the same jacket shape can be made using the 3D garment model of a jacket being worn by a body.

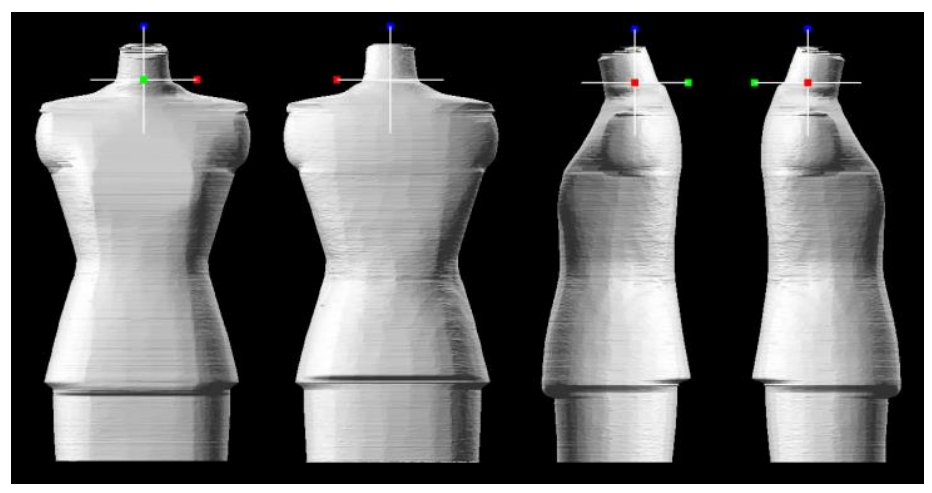
(a) front
(b) back
(c) left side
(d) right side

Figure 8. Basic garment model of Jacket1 

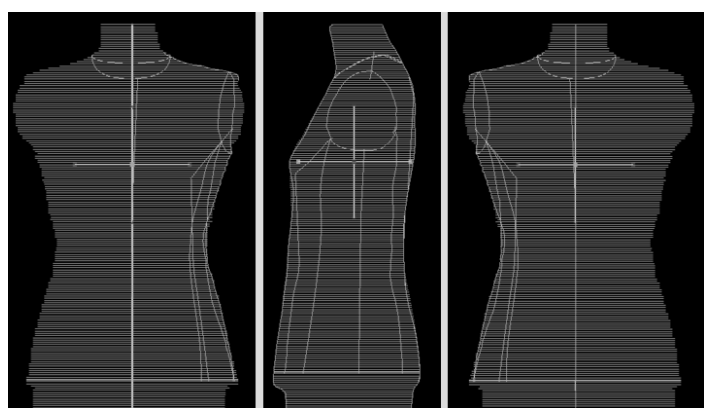

(a)

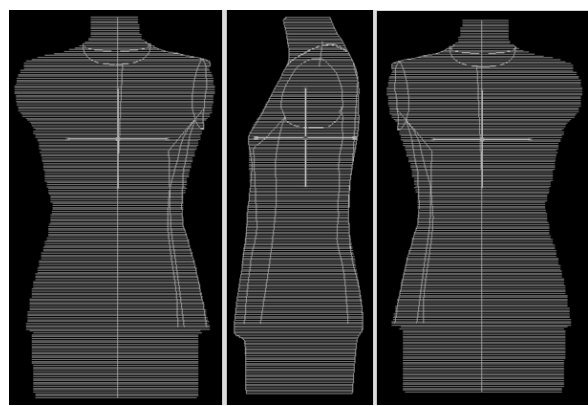

(b)

Figure 9. Configurations of the cutting lines; (a) original cutting lines and (b) cutting lines that differ from the original
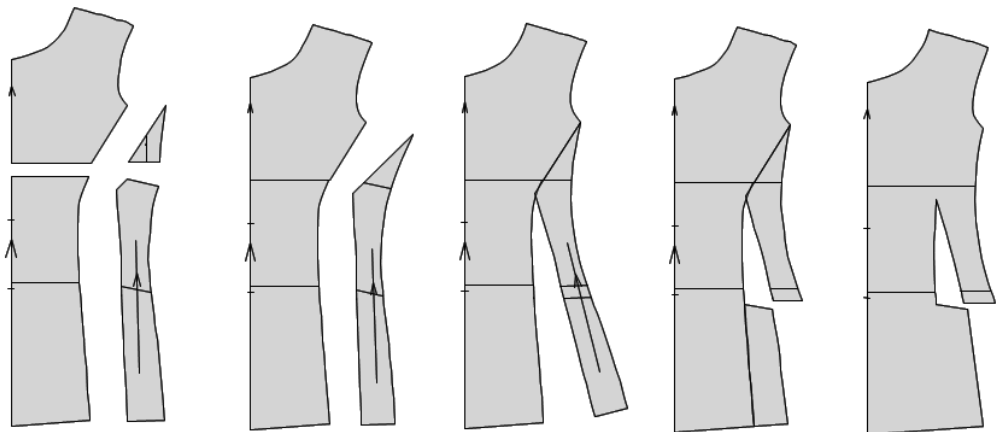

(a) separated pattern (b) combined pattern (c) cut patterns (d) final pattern

Figure 10. Segmentation and combination of the front pattern piece

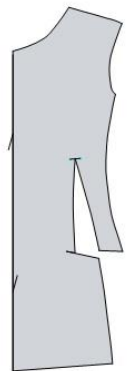

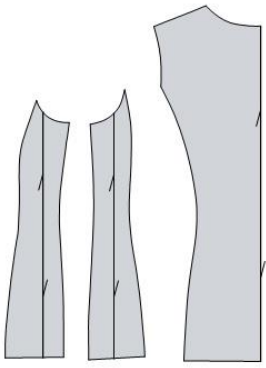

(a)

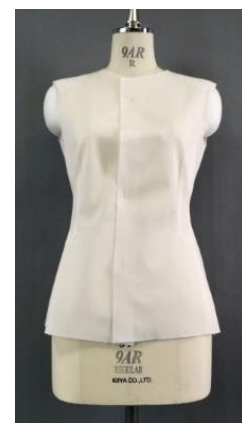

(b)

Figure 11. Patterns and made jacket with the same cutting lines (seam lines) for Jacket1
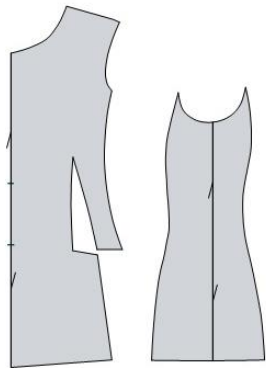

(a)

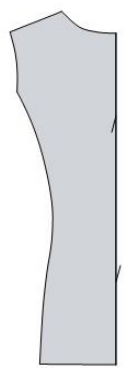

Figure 12. Patterns and made jacket with different cutting lines (seam lines) for Jacket1

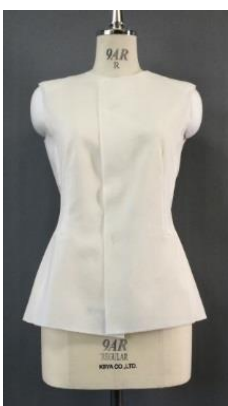

(b) 
Using our method, we measured the cross-sectional sizes of both the body and the original jacket and then calculated the multiplication factors in the front, back and lateral directions. The calculated multiplication factors are shown in Figure 13.

To confirm the magnification method, we made a size 9AR model and patterns using the multiplication factors and 9AR body. Figure 14 shows the reconstructed 9AR garment model and patterns. Figure 15 shows the reproduced jacket for 9AR. The silhouettes of the reconstructed jacket model in the front and side views in Figure 14 are similar to the basic garment model presented in Figure 8. Figure 22(a) shows the cross-sections of original and the reconstructed jacket models of Jacket1. The contour shapes of bust and hip of both models are similar, however the waist shapes are slightly different. The back of the original jacket was angular, whereas the back of the reconstructed model is round. This was due to an uneven allowance. The waist has a larger and more uneven allowance than the other parts, depending on direction. In our method, we took into account three directions and so the waist circumference became rounded. Despite this small difference in waist size, the overall shapes of the two models were similar. We also created two different garment models and pattern sizes, for 5AR and 13AR size bodies, using the multiplication factors of Jacket1. Figure 16 shows the garment model and patterns. Figures 17 and 18 show the jacket bodices for Jacket1. The jackets fitted on the bodies very well, indicating that the proposed method can be applied to make patterns for different size bodies.

We also obtained models and patterns of two different sizes of 5AR and 13AR bodies for Jacket2. The patterns are shown in Figure 19. Figures 20 and 21 show the made jacket bodices. Those jacket bodices also were fitted on the body well.

Figure 22(b) shows the cross-sections of original jacket and the reconstructed jacket model of Jacket2. They also show similar shape with each other. Moreover, the shapes of waist line were closer than ones of Jacket1. It was due to less allowance in waist area of Jacket2 than Jacket1. The waist allowances of Jacket 1 and Jacket 2 are $17.9 \mathrm{~cm}$ and $15.4 \mathrm{~cm}$, respectively (see Table 1 and 2). Thus, if the cross-section has less ease allowance, the constructed model is closer in shape to the original, but it is difficult to recapture the original shape if the ease allowance is large.

Figures 23 and 24 show the cross-sections of reconstructed garment model of Jacket1 and Jacket 2 for $5 \mathrm{AR}$ and 13AR size bodies. All the cross-section curves of models and jackets are almost the same respectively. We measured the sizes of all the models and jackets as shown in Table 2. Those sizes of made jackets agree with ones of the constructed garment models.

Consequently, we were able to make patterns in different sizes using the proposed method. Therefore, this method can be used as a size changing method especially for creating individualized garments.

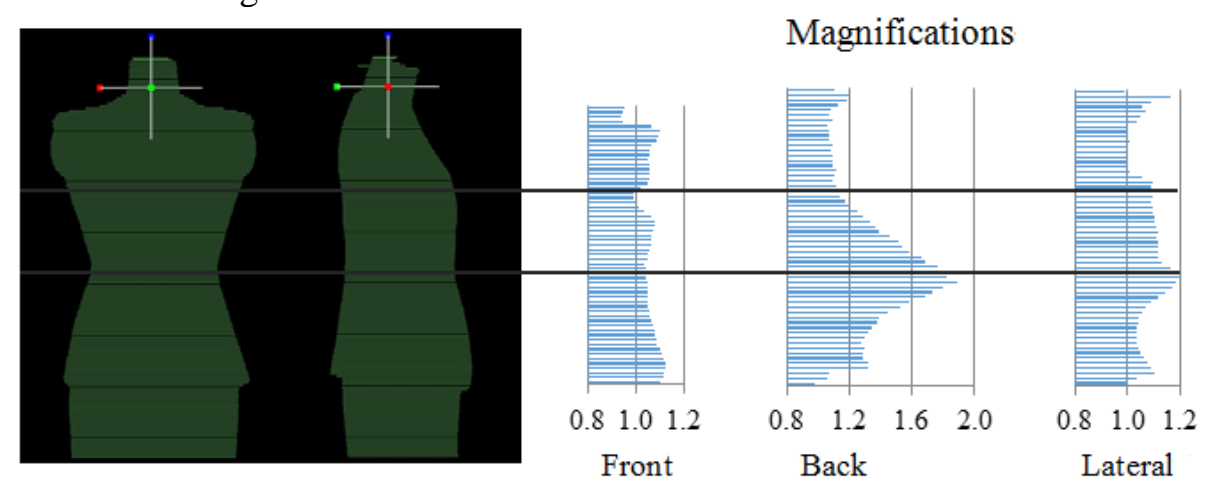

Figure 13. Multiplication factors in the front, back and lateral directions for Jacket1 


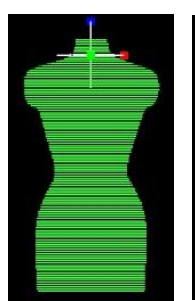

(a) 3D dada (b) front

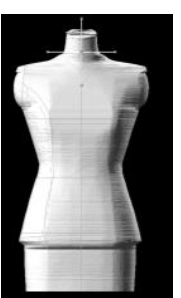

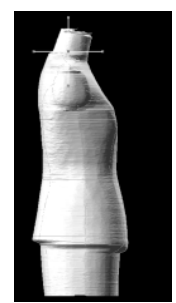

(c) side

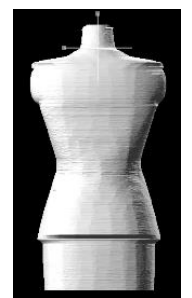

(d) back

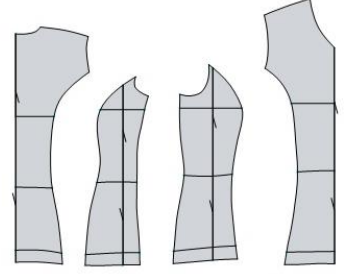

(e) patterns

Figure 14. Reconstructed jacket models and patterns of size 9AR for Jacket1

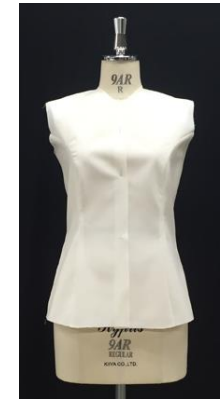

(a) front

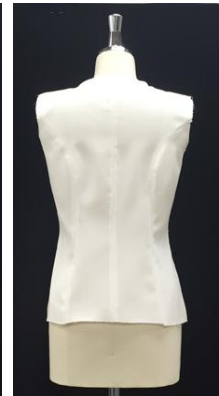

(b) back

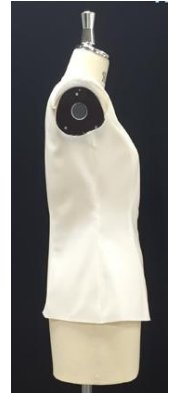

(c) side

Figure 15. Reproduced jacket of size 9AR for Jacket1

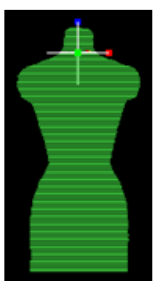

(a) 5AR model

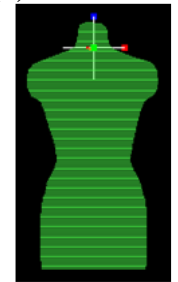

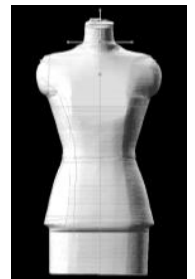

(b) 5 AR garment model

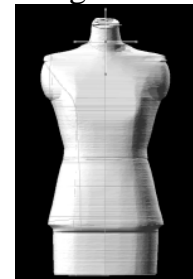

(d) 13AR model (e) 13AR garment model

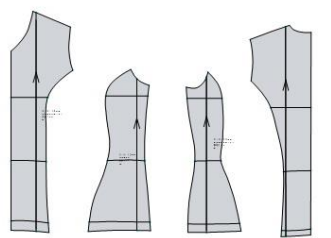

(c) 5AR Pattern

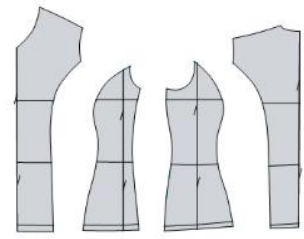

(f) 13AR Pattern

Figure 16. Constructed jacket models and patterns for Jacket1

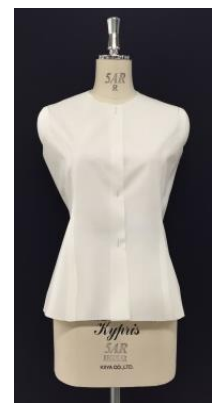

(a) front

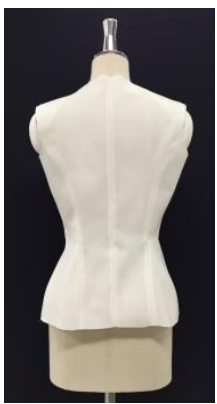

(b) back

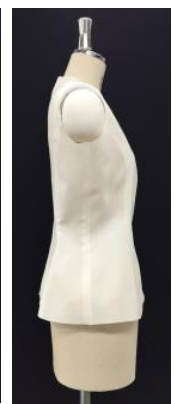

(c) side

Figure 17. Size 5AR jacket bodice of Jacket 1 


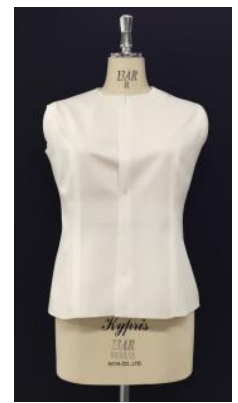

(a) front

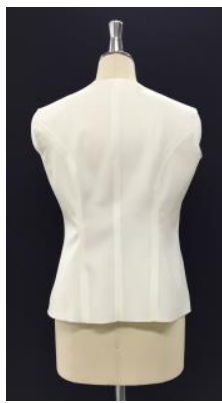

(b) back

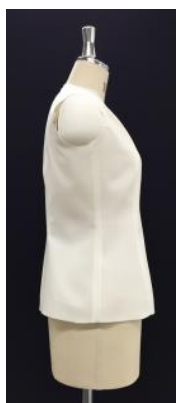

(c) side

Figure 18. Size 13AR jacket bodice of Jacket1

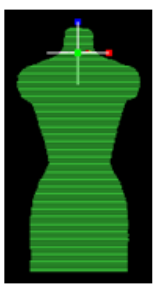

(a) 5AR model

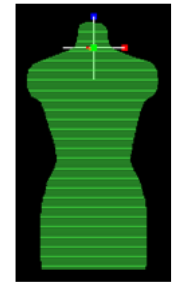

(d) 13AR model (e) 13AR garment model

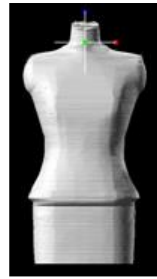

(b) 5AR garment model

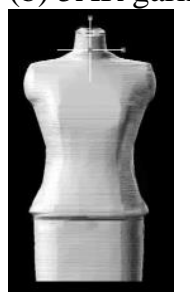

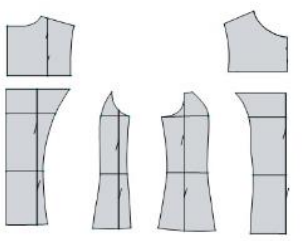

(c) 5AR Pattern

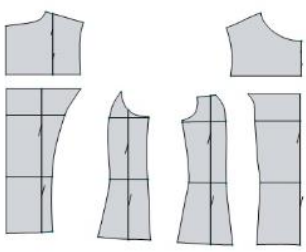

(f) 13AR Pattern

Figure 19. Constructed jacket models and patterns for Jacket2

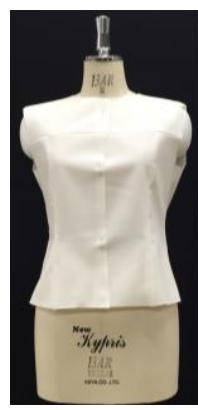

(a) front

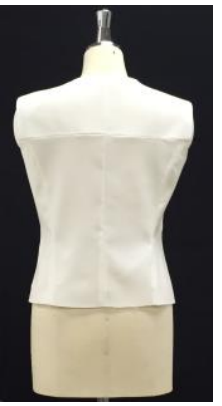

(b) back

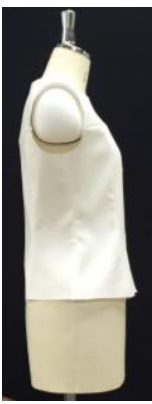

(c) side

Figure 20. Size 5AR jacket bodice of Jacket2
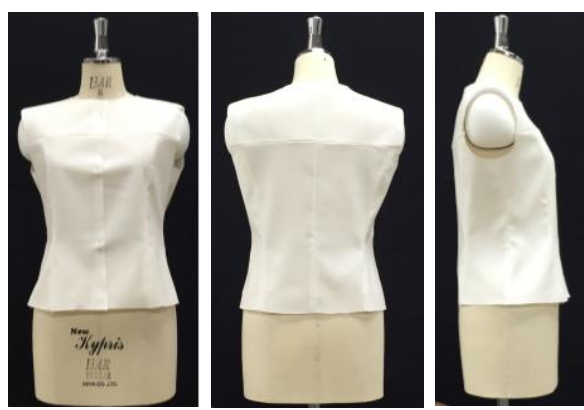

(a) front
(b) back
(c) side

Figure 21. Size 13AR jacket bodice of Jacket2

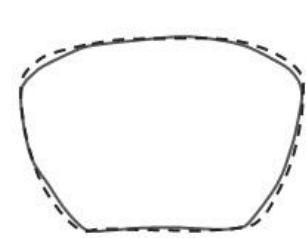

bust line

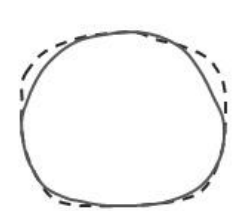

waist line

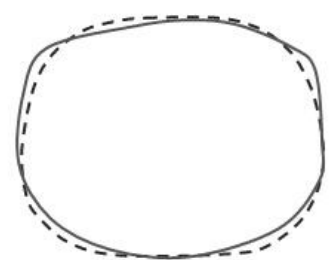

hip line

(a) Jacket1

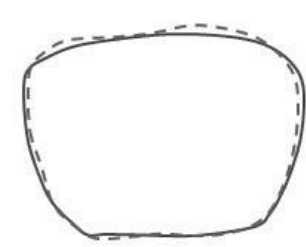

bust line

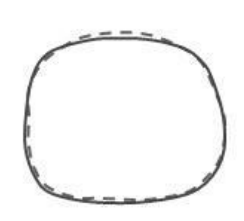

waist line

(b) Jacket2

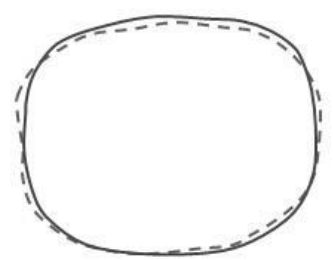

hip line

Reconstructed garment model

- - _ - _ $\quad$ Scanned original jacket (9AR)

Figure 22. Comparison of the cross-sections of the the constructed garment model and original jackets

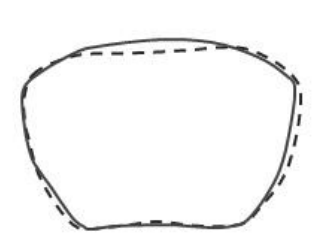

bust line

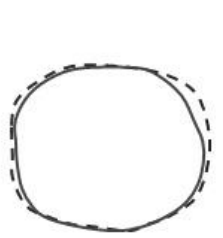

waist line

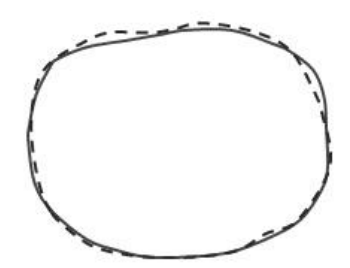

hip line

(a) $5 \mathrm{AR}$

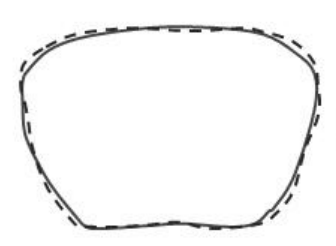

bust line

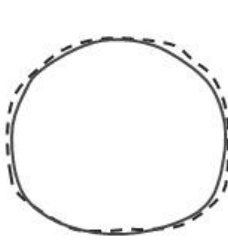

waist line

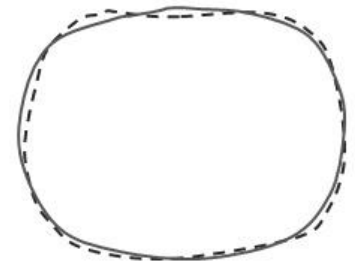

hip line

(b) $13 \mathrm{AR}$

Constructed garment model

- - _ - _ _ Scanned produced Jacket 1

Figure 23. Comparison of the cross-sections of the the constructed garment model and reproduced Jacket1. 


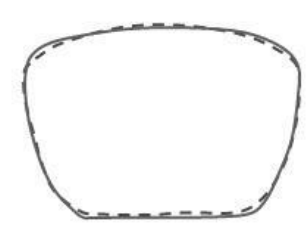

bust line

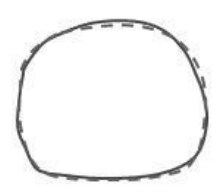

waist line

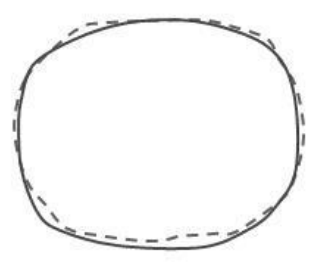

hip line

(a) $5 \mathrm{AR}$

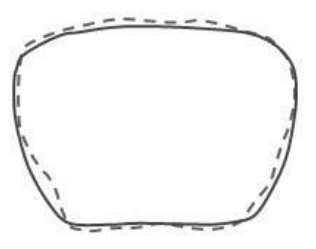

bust line

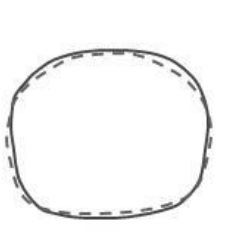

waist line

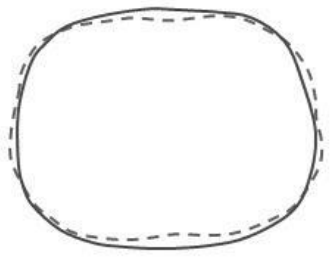

hip line

(b) $13 \mathrm{AR}$

Reconstructed garment model

- - _ - _ - Scanned produced Jacket2

Figure 24. Comparison of the cross-sections of the the constructed garment model and reproduced Jacket 2 .

Table 2. Measurements for each constructed garment model and made jacket (unit: $\mathrm{cm}$ )

\begin{tabular}{|l|l|c|c|c|c|c|c|}
\hline \multirow{2}{*}{ Jacket } & Body & \multicolumn{2}{|c|}{ Bust line } & \multicolumn{2}{c|}{ Waist line } & \multicolumn{2}{c|}{ Hip line } \\
\cline { 3 - 8 } & size & model & jacket & model & jacket & model & jacket \\
\hline \multirow{3}{*}{ Jacket1 } & 9AR & 94.0 & 94.3 & 80.5 & 80.9 & 104.9 & 103.1 \\
\cline { 2 - 8 } & 5AR & 85.3 & 85.9 & 68.9 & 70.4 & 99.1 & 99.9 \\
\cline { 2 - 8 } & 13AR & 98.0 & 97.9 & 83.4 & 84.4 & 107.9 & 107.4 \\
\hline \multirow{3}{*}{ Jacket2 } & 9AR & 93.6 & 94.0 & 78.8 & 78.4 & 99.1 & 98.2 \\
\cline { 2 - 8 } & 5AR & 83.6 & 84.2 & 67.9 & 68.6 & 92.9 & 94.6 \\
\cline { 2 - 8 } & 13AR & 96.6 & 97.2 & 82.9 & 83.6 & 102.3 & 102.5 \\
\hline
\end{tabular}

\section{Conclusions}

In this study we developed a new method for creating upper garment models suitable for 3D pattern-making. Our models take into account ease allowance and silhouette by using 3D scans of a garment model being worn by a body. A basic garment model of a jacket bodice was made using the method. To confirm the model validity, we used the 3D pattern-making system to produce bodice patterns with the same seam lines as the model. This manufactured jacket bodice possessed a shape similar to the original. Furthermore, we created a bodice pattern with seam lines that were different from the original ones, but that produced a bodice that also had a similar shape to the original. This confirmed that it was possible to make patterns that take into account ease allowance and silhouette by using a 3D scan of a garment model. Our method also can be used to make new patterns with different seam lines.

We also proposed a multiplication factor-based size-changing method. We calculated the size multiplication factors required between an actual garment worn on a body, and the body shape. This was done for cross-sections in the front, back, and lateral directions. Using the multiplication factors, two new models for different body sizes were constructed. We made patterns and garments from these models, and confirmed their accuracy. The garments fitted on the bodies 
very well, demonstrating that our method is applicable to patterns for different size bodies. Furthermore, appropriate patterns in different sizes for a different garment were also obtained.

This method can be substituted for the grading method when creating individually tailored garments.

\section{Acknowledgements}

This work was supported by JSPS (Japan Society for the Promotion of Science) KAKENHI under grant numbers 24220012.

\section{References}

Bigliani R., Eischen J.W., House D.H. and Breen D.E. (2000), "Collision Detection in Cloth Modeling" in "Cloth Modeling and Animation", A.K. Peters, Ltd, 199-205.

Bye, E., LaBat, K., McKinney, E. and Kim, D. E. (2008), "Optimized pattern grading", International Journal of Clothing Science and Technology, 20(2), 79-92.

Chan, A.P., Fan, J. and Yu, W.M. (2005), "Prediction of men's shirt pattern based on 3D body measurements", International Journal of Clothing Science and Technology, 17(2), 100-108.

Chen, Y., Zeng, X., Happiette, M., Bruniaux, P., Ng, R. and Yu, W. (2008), "A new method of ease allowance generation for personalization of garment design", International journal of clothing science and technology, 20(3), 161-173.

Cho, Y.S., Okada, N., Park, H.J., Takatera, M., Inui, S. and Shimizu, Y. (2005), “An interactive body model for individual pattern making", International Journal of Clothing Science and Technology, 17(2), 91-99.

Cho, Y.S., Komatsu, T., Inui, S., Takatera, M., Shimizu, Y. and Park, H. (2006a), "Individual pattern making using computerized draping method for clothing", Textile research journal, 76(8), 646-654.

Cho, Y.S., Komatsu, T., Takatera, M., Inui, S., Shimizu, Y. and Park, H. (2006b), "Posture and depth adjustable 3D body model for individual pattern making", International Journal of Clothing Science and Technology, 18(2), 96-107.

Cho, Y.S., Tsuchiya, K., Takatera, M., Inui, S., Park, H. and Shimizu, Y. (2010), "Computerized pattern making focus on fitting to 3D human body shapes", International Journal of Clothing Science and Technology, 22(1), 16-24.

Daanen, H. and Hong, S. A. (2008), "Made-to-measure pattern development based on 3D whole body scans", International Journal of Clothing Science and Technology, 20(1), 15-25.

Joseph-Armstrong H. (2009) Patternmaking for Fashion Design 5th edition, Prentice Hall, New Jersey, 68-97.

Digital Fashion Ltd. (2014) digital fashion ltd. Available from: http://www.digitalfashion.jp [Accessed: 2nd Dec. 2014].

Hlaing, E.C., Krzywinski, S. and Roedel, H. (2013), "Garment prototyping based on scalable virtual female bodies", International Journal of Clothing Science and Technology, 25(3), 184-197.

Imaoka H., Shibuya A. and Aisaka N., (1989), "Automatic paper pattern making using mechanical development method of a curved surface on a plane surface". Sen'i Gakkaishi, 45(10), 427-434.

Jeong, Y., Hong, K. and Kim, S. J. (2006), "3D pattern construction and its application to tightfitting garments for comfortable pressure sensation", Fibers and Polymers, 7(2), 195-202.

Kim, S., Jeong, Y., Lee, Y. and Hong, K. (2010), "3D pattern development of tight-fitting dress for an asymmetrical female manikin", Fibers and Polymers, 11(1), 142-146.

Kim, S. and Park, C.K. (2004), "Parametric body model generation for garment drape simulation", Fibers and Polymers, 5(1), 12-18. 
Mahnic, M. and Petrak, S. (2013), "Investigation of the Fit of Computer-based Parametric Garment Prototypes", Journal of Fiber Bioengineering and Informatics, 6(1), 51-61.

Takahashi, Y., Sofue, A., Kim, K.O. and Takatera, M. (2013), "Automatic 3D pattern making of fitted upper garments considering individuals' figures and shear limit of fabrics", International Journal of Affective Engineering, 12(2), 169-175.

Takatera, M. and Kim, K.O. (2013), "Improvement of Individualized Pattern Making Using Surface Flattening Technique", Journal of Fiber Bioengineering and informatics, 6(4), 467480.

Takatera, M., Sofue, A., Kim, K.O. and Inui, S. (2010), "Automatic 3D pattern making of basic pattern for upper garments", Proceedings of the 39th Textile Research Symposium, December 6-18, 2010, Delhi, India, 317-323.

Troynikov, O. and Ashayeri, E. (2011), "3D body scanning method for close-fitting garments in sport and medical applications", In R. Mitchell (Ed.),HFESA 47th Annual Conference, Crows

Thomassey, S. and Bruniaux, P. (2013), A template of ease allowance for garments based on a 3d reverse methodology, International Journal of Industrial Ergonomics, 43(5), 406-416.

Wang, Z.H. and Newton, E. (2007), "Modeling of cross-sectional shape for women's jacket design", Sen'i Gakkaishi, 63(4), 87-96.

Wang, Y. N. and Huang, H. Q. (2011), "Three dimensional pattern grading based on deformable body features and 3D developable surface", Journal of Fiber Bioengineering and Informatics, 4(2), 115-128.

Watanabe Y. (1999), "Ordering your cloth to fit yourself", The Journal of the Institute of Electronics, Information and Communication Engineers, 82(4), 404-411.

Yang.Y.C. and Zhang.W.Y. (2007) "Prototype garment pattern flattening based on individual 3D virtual dummy". International Journal of Clothing Science and Technology, 19(5), 334-348.

Yuka \& Alpha Apparel Solution (2014) Yuka And Alpha Co.,Ltd. Available from: http://yukaalpha.com [Accessed: 2nd Dec. 2014].

Zhang M., Kamijo M., Takatera M., Furukawa T., \& Shimizu Y. (2000), “An interactive pattern making and 3D simulation system of collars". Kansei Engineering International, 1(4), 1-10. 